\title{
Erratum to: A novel dynamic network data replication scheme based on historical access record and proactive deletion
}

\author{
Zhe Wang • Tao Li • Naixue Xiong · Yi Pan
}

Published online: 21 December 2011

(C) Springer Science+Business Media, LLC 2011

\author{
Erratum to: J Supercomput \\ DOI 10.1007/s11227-011-0708-z
}

In the above mentioned article the authors wish to publish the following acknowledgment section.

This work was supported by National Natural Science Foundation of China (Grant No. 60873246 and Grant No. 61173159) and the Cultivation Fund of the Key Scientific and Technical Innovation Project by Ministry of Education of China under Grant No. 708075.

The online version of the original article can be found under doi:10.1007/s11227-011-0708-z.

Z. Wang · T. Li (凶)

Dept. of Computer Science, Sichuan University, Chengdu, China

e-mail: litao@scu.edu.cn

Z. Wang

e-mail: wangz84626@gmail.com

N. Xiong · Y. Pan

Dept. of Computer Science, Georgia State University, Atlanta, USA

N. Xiong

e-mail: nxiong@cs.gsu.edu

Y. Pan

e-mail: pan@cs.gsu.edu 